\title{
Concepciones de niño y modos de relación de cuidadores y profesionales con niños institucionalizados, Colombia
}

\author{
José Eduardo Sánchez-Reyes, Mg. \\ Profesor Universidad Icesi, Colombia* \\ Jackeline Cantor-Jiménez, Mg. \\ Profesora Universidad Icesi, Colombia**
}

\author{
Ximena Castro-Sardi, Mg. \\ Profesora Universidad Icesi, Colombia*** \\ Yamileth Bolaños, Mg. \\ Profesora Universidad Icesi, Colombia****
}

\section{Resumen (descriptivo)}

Este artículo explora las diferentes concepciones de niño y las formas de relación que tienen los profesionales y los cuidadores de niños y adolescentes en una institución de protección en Colombia. Se parte del supuesto que tanto las concepciones de sujeto, como los modos de relación orientan las prácticas de los cuidadores en la institución. Durante el estudio se analizan 13 entrevistas realizadas a cuidadores y profesionales de la institución de protección, observaciones participantes y documentos. Emergen tres concepciones de niño: el niño como hijo de Bienestar, el niño resiliente, y el niño en carencia. Los profesionales y cuidadores se encuentran en tensión entre su labor profesional de restitución de derechos y la labor de crianza tradicionalmente desempeñada por las familias.

\section{Palabras clave}

Bienestar de la infancia, derechos del niño, institucionalización.

\section{Tesauro}

Tesauro de Ciencias Sociales de la Unesco.
Para citar este artículo

Sánchez-Reyes, J. E., Cantor-Jiménez, J., Castro-Sardi, X., \& Bolaños, Y. (2019). Concepciones de niño y modos de relación de cuidadores y profesionales con niños institucionalizados, Colombia. Revista Latinoamericana de Ciencias Sociales, Niñez y Juventud, 17(2), 1-24 doi:

10.11600/1692715x.17209

\section{Historial}

Recibido: 30.11.2018

Aceptado: 21.01.2019

Publicado: 30.06 .2019

Información artículo

Este artículo se deriva de la investigación «Caracterización del Modelo de Intervención de un Internado del ICBF en Cali» adelantado por el Departamento de Estudios Psicológicos de la Universidad Icesi, Cali (Colombia). La investigación fue financiada en el marco de la convocatoria interna 2016 de la Universidad Icesi y fue desarrollada por los autores de septiembre a diciembre de 2016. Área: Ciencias Sociales. Subárea: Interdisciplinaria. 


\section{Conceptions of children and ways of relating between caregivers and professionals and institutionalized children, Colombia}

\section{Abstract (descriptive)}

This article explores the different conceptions of children and the ways of relating between caregivers and professionals and children and adolescents in a protection institution in Colombia. It is assumed that both the conceptions of children and the different ways that adults have of relating to them guide the caregivers' practices in the institution. During the study, 13 interviews with caregivers and professionals of from protection institution were conducted along with participant observation and documents analysis. Three conceptions of the child subject emerged: the child as a son/daughter of the welfare system, the resilient child and the child in need. Professionals and caregivers face tension between their professional work to facilitate the restitution of these children's rights and the parenting role traditionally carried out by families.

Keywords

Child welfare, rights of the child, institutionalization.

\section{Concepções de infância e modos de relação entre cuidadores e profissionais com a infância institucionalizada, Colômbia}

\section{Resumo (descritivo)}

Este artigo considera as diferentes concepções de crianças e os tipos de relacionamento que profissionais e cuidadores de crianças e adolescentes têm em uma instituição de proteção na Colômbia. Parte-se do suposto que tanto as concepções de sujeito, como os modos de relacionamento orientam as práticas dos cuidadores em uma instituição. Neste estudo, analisam-se 13 entrevistas realizadas a cuidadores e profissionais da instituição de proteção, observações participantes e documentos. Emergem três concepções de criança: a criança como filho de bem-estar, a criança resiliente, e a criança em carência. Os profissionais e cuidadores encontram-se em tensão entre o seu labor profissional de restituição de direitos e o labor de criação tradicionalmente desempenhado pelas famílias.

\section{Palavras-chave}

Bem-estar da infância; direitos da criança; institucionalização.

\section{Información autores}

[^] Psicólogo y Magíster en Psicología de la Universidad del Valle (Colombia). Director del programa de Psicología en la universidad Icesi, Cali, Colombia y, docente del Departamento de Estudios Psicológicos. Orcid:

0000-0002-4580-1894. Índice H5: 1. Correo electrónico: jesanchez@icesi.edu.co

[**] Psicóloga y Magíster en Psicología de la Universidad del Valle (Colombia). Profesora del Departamento de Estudios Psicológicos de la Universidad Icesi, Cali, Colombia. Orcid: 0000-0002-6339-8701. Índice H5: 1. Correo electrónico: jcantor@icesi.edu.co

[***] Psicóloga de la Universidad de los Andes (Colombia) con maestría en Estudios Psicoanalíticos de la New School University (EEUU) y maestría en Investigación en Psicoanálisis de la Universidad París VIII (Francia); en esta última es candidata a Doctora en Psicoanálisis. Profesora del Departamento de Estudios Psicológicos de la Universidad Icesi, Cali. Orcid: 0000-0002-0740-9293. Índice H5: 1. Correo electrónico: xcastro@icesi.edu.co

[***] Psicóloga y Magíster en Estudios Sociales y Políticos de la Universidad Icesi (Colombia). Profesora del Departamento de Estudios Psicológicos de la Universidad Icesi, Cali, Colombia. Orcid: 0000-0003-0863-6559. Índice H5: 0. Correo electrónico: bmyamileth@gmail.com 


\section{Introducción}

La problemática de niños, niñas y adolescentes que no cuentan con el cuidado de sus familias a lo largo de su crianza tiene como alternativa de solución la institucionalización en centros de acogimiento residencial. Estudios sobre el tema en Latinoamérica han expresado la necesidad de que los Estados incluyan políticas de fortalecimiento familiar e implementen programas efectivos de protección a la familia, de manera que consideren opciones diferentes a la institucionalización (Fernández-Daza, 2018; Palummo, 2013). A través de fuentes diversas, Palummo (2013) estima que existen más de 240000 niños, niñas y adolescentes (NNA en adelante) institucionalizados en Latinoamérica. En Colombia se estima que la cifra es de 12925 niños (Palummo, 2013).

En relación a la existencia de NNA abandonados o con derechos vulnerados en sus grupos familiares, la institucionalización aparece como una opción posible y transitoria - aunque no la más deseable-para lograr el restablecimiento de los derechos. Por ejemplo, en el año 2010 algunos países latinoamericanos reportaron tener un total de 48536 ingresos de niños a instituciones de cuidado y 22251 egresos en el mismo año (Palummo, 2013). Lo anterior sugiere que hay un número importante de niños que permanecen sin otras alternativas frente a la institucionalización, como la adopción (Instituto Colombiano de Bienestar Familiar, 2015) ${ }^{1}$.

El estudio de NNA en instituciones de protección se ha centrado en la importancia del entorno para su desarrollo, teniendo como referente el desarrollo de los niños que viven con sus familias. Algunas investigaciones han realizado comparaciones entre NNA que se encuentran en ambientes institucionales frente a NNA en contextos familiares (Deambrosio, Gutiérrez de Vázquez, Arán-Filippetti, \& Román, 2018; Fernández-Daza

\footnotetext{
${ }^{1}$ En 2015 el Instituto Colombiano de Bienestar Familiar expresó que existían 4850 niños y niñas con necesidades especiales (es decir, pertenecientes a grupos de hermanos, mayores de 8 años o con algún tipo de discapacidad o problema de salud permanente) en espera de ser adoptados, frente a 368 solicitudes de familias adoptantes colombianas.
} 
\& Fernández-Parra, 2013; Smyke et al., 2007), al igual que estudios de carácter exploratorio (Silva da Cruz, Dias, \& da Silva, 2014) y han encontrado en déficit a los primeros frente a los segundos, en aspectos como el comportamiento, el rendimiento escolar, el crecimiento físico, el lenguaje y el desarrollo cognitivo.

Otras perspectivas han destacado el agenciamiento de los sujetos en las instituciones de protección para resignificar sus experiencias e iniciar un proceso resiliente (Obando, Villalobos, \& Arango, 2010). En la mayoría de los estudios mencionados se resalta el papel de los cuidadores en los procesos de desarrollo de NNA y de sus familias para el establecimiento de vínculos significativos y de confianza que propicien el desarrollo de los menores y su socialización; además de la necesidad de ahondar en estas temáticas (Amar, Tirado, \& Barreneche, 2007; Gabatz, Schwartz, Milbrath, Carvalho, Lange, \& Soares, 2018; Obando et al., 2010).

Teniendo en cuenta lo anterior, el objetivo central de este artículo es explorar las diferentes concepciones de niño ${ }^{2}$ las formas de relación que tienen los cuidadores y los profesionales de apoyo ${ }^{3}$ con los niños en una institución de protección ${ }^{4}$ que acoge NNA entre los 7 y los 24 años, ubicada en la ciudad de Cali. Se plantea que tanto las concepciones de sujeto como los modos de relación orientan las prácticas de los cuidadores.

A continuación se presenta un breve recorrido en relación a la transformación de la asistencia social en Latinoamérica, el cambio en la noción de niñez y la labor de crianza asumida en entornos no familiares. Esto con el propósito de ofrecer un contexto histórico que permita comprender las prácticas actuales de restablecimiento de derechos.

\section{Transformación en la asistencia social en Latinoamérica}

Durante los siglos XIX y XX se presenta en Colombia y Latinoamérica la transformación de la asistencia social de caridad y beneficencia a una de asistencia pública y acción social. El apoyo desde diversos sectores a las necesidades sociales durante esta época se re-

2 El estudio se desarrolla en una institución que acoge únicamente a niños varones.

3 En el artículo se presentan de manera diferenciada las voces de cuidadores y profesionales de apoyo. Los cuidadores en algunos casos son también profesionales (aunque la mayoría cuenta solamente con estudios técnicos o tecnológicos), siendo una de las diferencias entre ellos el que los cuidadores están en turnos; lo anterior implica que, en algunos casos, deben pasar la noche con los NNA, mientras que los profesionales cumplen funciones durante la jornada laboral regular.

4 Las instituciones prestadoras de servicios de protección a NNA en Colombia están bajo potestad del Instituto Colombiano de Bienestar Familiar (en adelante ICBF). 
lacionó con la condición de pobreza (Paz, 2014), y la realización de dichas prácticas fue liderada por la Iglesia católica ${ }^{5}$, además de profesionales de la salud y voluntarios provenientes principalmente de clases sociales media y alta (Castro, 2008; Paz, 2014).

Las prácticas de caridad fueron lugar de origen para la consolidación de estrategias que se entendían en Colombia como el trabajo social desinteresado y abnegado. Estas prácticas permitían que los católicos, desde su perspectiva, se perfeccionaran espiritualmente, mientras que las donaciones tenían como fin atraer la atención de Dios y posibilitar la salvación del alma.

En la mitad del siglo XIX la noción de caridad es puesta en discusión por la secularización del Estado, y se da paso al término beneficencia, que designa el acto de dar como un deber ciudadano o estatal, desligado de toda connotación religiosa (Castro, 2008).

Desde principios del siglo XX se introduce lo social en la agenda del gobierno colombiano a través del concepto de asistencia pública. El Estado asigna ayudas a instituciones laicas y religiosas que se dedican a la beneficencia, priorizando la ayuda destinada a la educación, y se crean orfanatos (Castro, 2008).

En la historia de Colombia se destaca la participación tanto del gobierno como de los partidos políticos en la creación de entidades sin ánimo de lucro que prestan servicios sociales; sin embargo, desde la década de los ochentas y luego, con la Constitución de 1991, se introdujeron cambios tendientes a la descentralización para permitir una mayor autonomía de las organizaciones del tercer sector frente a entidades estatales (Villar, 2001).

\section{Concepción de niñez y transformación legislativa}

Según Ariès (1987) la concepción de infancia actual es una construcción que inicia en la época medieval, cuando había una indiferencia generalizada frente a la niñez. Solo después del control de la natalidad desaparecerá esta indiferencia, hasta ubicar los problemas de la infancia en el centro de la agenda pública.

Desde el campo del derecho existen dos ideologías relacionadas con la niñez. La doctrina de la situación irregular, que inicia en el siglo XIX en Estados Unidos y Europa, y se presenta en Latinoamérica como doctrina de protección-represión del Estado hacia niñas y niños excluidos de la sociedad. En contraposición a la anterior, surge la doctrina de la protección integral, a finales del siglo XX. Esta se basa en la Declaración Universal de los

\footnotetext{
5 Colombia reconoció al catolicismo como religión oficial hasta 1991.
} 
Derechos del Niño que establece una concepción de niño y niña como sujetos plenos de derechos (Jiménez, 2009). En la actualidad, la mayor parte de las constituciones políticas latinoamericanas reconocen como fundamentales los derechos de los niños y las niñas, en consonancia con la ratificación y vigencia de la Convención Internacional de los Derechos del Niño (en adelante CIDN) (Nogueira, 2017).

Por su parte, Colombia ratifica la adherencia a la CIDN en 1991, pero solo hasta el 2006 actualiza sus leyes con la promulgación del Código de la Infancia y la Adolescencia, en consonancia con la CIDN (Castrillón, 2008).

Llobet (2011) encuentra dos problemas relacionados con la implementación de la CIDN: 1) las y los adultos toman la voz para hablar desde su perspectiva sobre la niñez, sin permitir que niñas y niños se representan a sí mismos; 2) la CIDN llega a Latinoamérica cuando predominan las reformas neoliberales que impactan la concepción del Estado. Esto da paso a una mirada individualista de las problemáticas de la niñez que olvida el contexto en el cual suceden. Para Villalta (2013) el hecho de garantizar el interés superior de niños y niñas por parte de dispositivos jurídico-burocráticos, hace parte de una necesidad de regularizar relaciones familiares, formas adecuadas de crianza y valores de la paternidad y la maternidad. No obstante, dicha regulación puede ser analizada desde diversos sentidos, como medida de control y como medida benéfica.

De acuerdo con Llobet (2011), el concepto de niño o niña como sujeto de derechos se asemeja a un significante vacío que permite que se adhieran a él definiciones de niñez constituidas por discursos traídos de la psicología individual. Desde esta perspectiva, los traumas son comprendidos como estructurantes de la subjetividad y traen consecuencias futuras, dejando de lado otros aspectos de la constitución subjetiva y las relaciones sociales del sujeto en el presente. Sin embargo, Llobet señala que los sentidos de los derechos son producidos por los actores, de ahí que puedan cambiar. Para la autora, aunque hay discursos sobre los derechos de la niñez que excluyen a grupos vulnerables; en la ciudadanía existe el acto performativo: los niños y niñas pueden usar el discurso sobre sus derechos.

En este sentido, se han encontrado usos de la identidad estratégicos ${ }^{6}$ (Sánchez-Reyes, 2015) porque ciertos individuos pertenecientes a grupos minoritarios hacen un uso del discurso que reconoce las desigualdades respecto a grupos minoritarios; esto les permite acceder a derechos y garantías para su beneficio individual y colectivo, frente al resto de la población, fortaleciendo su identidad.

${ }^{6}$ Estratégico no denota intencionalidad negativa; alude a la conciencia y uso de características identitarias. 


\section{Familia e instituciones}

En la actualidad existe una visión crítica respecto a la institucionalización de los NNA, dado que no garantiza su derecho a vivir en familia (Durán \& Valoyes, 2009). Rodrigo y Palacios (1998) señalan que dentro de una institución de protección la relación que se establece entre los NNA y cuidadores no tiene el carácter de compromiso personal ni la carga emocional existente en la familia. Durán y Valoyes (2009) establecen que el personal que atiende las necesidades de los NNA en el restablecimiento de sus derechos, no puede asimilarse a las figuras familiares. Además, la creación de lazos afectivos con los NNA dentro de una institución de protección es entorpecida por el número de los mismos, la rotación del personal, la duración de la institucionalización, la falta de celeridad en los procesos administrativos, entre otros.

La función primordial de la familia es la integración sociocultural de su descendencia (Jorge \& Gonzáles, 2017; Rodrigo \& Palacios, 1998). Lo anterior implica que los niños y las niñas al llegar al mundo requieren, para humanizarse, ser acogidos por otros; en este sentido es fundamental el papel de la familia como filtro cultural. Desde la legislación internacional se reconoce el derecho primordial de niños y niñas a tener una familia porque esta se entiende como «el vínculo interpersonal y medio natural para el desarrollo personal integral de todos sus integrantes y en particular de los niños» (Comisión Interamericana de Derechos Humanos, 2013, p. 17).

No obstante, la familia moderna es una construcción social que aparece en el siglo XVII (Levin, 2000) y solo es hasta finales del siglo XIX que en Estados Unidos y Europa surge el interés estatal por proteger a la niñez de maltratos, estableciéndose leyes y castigos morales a las familias en casos de negligencia parental (Grinberg, 2015).

El proyecto de la modernidad adscribió el cuidado y la educación de los niños y niñas a instituciones como la escuela (espacio público) y la familia nuclear (espacio privado) que, a su vez, conllevaron a una diferenciación entre saberes especializados frente a saberes derivados de lazos de proximidad. Esta distinción ha legitimado socialmente la adscripción de la familia al orden de lo natural y lo moral, mientras que se la ha desvinculado de lo colectivo y de las relaciones de poder (Santillán, 2009).

Actualmente las distinciones entre lo público y lo privado se han redefinido, así como la paternidad y maternidad contemporáneas, principalmente por la transformación de identidades masculinas y femeninas, los cambios económicos en la sociedad contemporánea y las transformaciones en las relaciones entre hombres y mujeres (Micolta, 
2011), lo que ha permitido la flexibilidad en los roles tradicionales al interior de las familias y la aceptación gradual de nuevos arreglos familiares.

Teniendo en cuenta lo anterior, aunque no se desconoce el papel fundamental que cumplen la familia y las instituciones de educación en la socialización, se resalta que no son los únicos espacios que asumen esta tarea (Santillán, 2009).

Por tanto, esta investigación se desarrolla en una institución que ofrece servicios de cuidado y atención integral a NNA entre los 7 y 24 años, en situación de vulneración de derechos, bajo contratación del Estado. El objetivo de esta investigación es explorar las diferentes concepciones de niño y las formas de relación que tienen los cuidadores y los profesionales con los niños en esta institución.

Las categorías concepciones de niño y estilos de relación entre cuidadores/profesionales con los niños en programas estatales o instituciones destinadas a la crianza de niños y niñas han sido señaladas en estudios similares como aspectos relevantes a incluir en agendas de investigación que retomen variables culturales y sociales (Amar et al., 2007). Siguiendo la misma línea, esta investigación adopta las categorías mencionadas y asume un compromiso social en la medida que aporta en la deconstrucción del discurso adulto sobre niños y niñas abandonados que los ubica exclusivamente en una posición de víctimas (Obando et al., 2010).

Para explorar estas concepciones y estilos de relación se optó un enfoque de investigación social cualitativo, que tuvo en cuenta estrategias metodológicas - implementadas en estudios similares como los realizados por Durán y Valoyes (2009) y Otálvaro, Peñaranda, Bastidas, Torres y Trujillo (2016) - como el análisis documental, la realización de entrevistas semiestructuradas con actores que trabajan con población de NNA sin cuidado parental y la observación participante.

\section{Método}

Este estudio parte de una perspectiva interpretativa y adopta el enfoque de la investigación social cualitativa. Atiende por tanto a los procesos sociales e históricos buscando reconocer la singularidad de las experiencias subjetivas (Crotty, 2006; Galeano, 2004).

Adicionalmente, este estudio se basa en los presupuestos de la teoría fundamentada, en la medida que prevalece la emergencia del conocimiento a través de la información del campo. Específicamente, la teoría fundamentada es un método que permite construir teo- 
ría «derivada de datos recopilados de manera sistemática y analizados por medio de un proceso de investigación» (Strauss \& Corbin, 2002, p. 21). Si bien la pretensión no es la de construcción de teoría, se hace uso de este método para describir las formas de relación que establecen cuidadores y profesionales con niños en una institución de protección.

\section{Características institucionales}

Esta investigación se desarrolló en una institución de protección ubicada en Cali (Colombia), fundada en 1959 como hogar de beneficencia. Actualmente la institución presta sus servicios al Instituto Colombiano de Bienestar Familiar (ICBF) —organismo estatal protector de los derechos de NNA en Colombia- bajo la modalidad de internado, 24 horas del día, 7 días de la semana. La institución cuenta con 97 beneficiarios entre los 7 y 24 años, 55 de ellos declarados en adoptabilidad7 y 42 en espera para retornar a su medio familiar o para ser declarados en adoptabilidad.

\section{Participantes}

Se trabajó con una muestra de 13 trabajadores de la institución de protección ( 7 mujeres y 6 hombres), de los cuales, 6 eran cuidadores y 7 profesionales ( 2 de ellos cumplían además funciones de dirección administrativa y orientación a los NNA), todos con al menos 1 año de experiencia laboral en la institución. Entre los profesionales, los directivos tenían formación en carreras administrativas; los otros profesionales de apoyo tenían estudios en trabajo social, psicología y salud, y atendían a los NNA en horario laboral. Los cuidadores contaban con formación en pedagogía, artes o deporte, y acompañaban a NNA en sus actividades cotidianas por turnos durante el día o la noche.

\section{Selección de participantes}

La investigación surge de un proceso de reflexión, posterior a un pedido de asesoría por parte de los directivos de la institución que buscaba recomendaciones para mejorar sus procesos y prácticas de servicio social. Se realizó un muestreo por conveniencia, por lo cual se entrevistó a las personas implicadas directamente en la atención de los NNA, entre ellos directivos con capacidad de decisión y con una función de orientación cotidiana. Se entrevistó a los siete profesionales (cuatro profesionales de apoyo psicosocial, un

\footnotetext{
${ }^{7}$ La declaratoria de adoptabilidad es una medida de restitución de derechos de la niñez y la adolescencia confirmada por un juez; esta busca garantizar el derecho de niños y niñas a tener una familia.
} 
profesional de apoyo en salud y dos con funciones directivas y de apoyo) y a seis cuidadores permanentes de la institución. Todos aceptaron participar voluntariamente.

\section{Procedimiento}

Inicialmente se hicieron notas de campo y observaciones participantes en la institución, durante tres meses. Posteriormente, se definieron las categorías de las entrevistas hechas a los actores de la institución. Las entrevistas duraron aproximadamente 60 minutos, siguiendo un curso narrativo.

De forma paralela se seleccionaron y analizaron aquellas fuentes secundarias vinculadas a la protección de los NNA, como archivos históricos de la institución y documentos relacionados directamente con el servicio en los que se describen políticas y protocolos de atención.

Se usó el software Atlas.ti ${ }^{\mathrm{TM}}$, para analizar y codificar los datos tomando como base la teoría fundamentada. Este software permite que la exploración y codificación de los datos se pueda desarrollar por medio de la lectura de fragmentos de texto, que se complementan a su vez con notas de campo de los investigadores (San Martín, 2014).

\section{Categorías de la entrevista}

Se identificaron 11 categorías: trayectoria laboral, historia de la institución, concepción del niño o adolescente que vive en la institución, significado de la institución, objetivo de la intervención con los NNA, relaciones al interior de la institución, prácticas laborales, dificultades para la labor desempeñada, necesidades de los NNA de la institución, mejoramiento de la institución y programas institucionales. Este artículo se centra en el análisis de tres: la concepción de sujeto, las prácticas institucionales de cuidado y las relaciones dentro de la institución. Cada una de estas categorías se dividió en subcategorías emergentes, con ayuda del software Atlas.ti, a excepción de la categoría «prácticas institucionales de cuidado» que se acompañó de observaciones de campo (tabla 1).

Durante el trabajo de campo emergieron tres concepciones de niño 8 enunciadas por las y los participantes: hijos de Bienestar, niños en carencia, niños resilientes. A través de estas se presentan los resultados y se recurre a la voz de los participantes para ejemplificarlas.

8 Por concepción de niño se entiende concepción de niño y de adolescente. 


\section{Tabla 1}

\section{Categorías iniciales}

Categorías iniciales

Concepción de sujeto: forma de entender y de nombrar a niños y adolescentes por parte de cuidadores y profesionales.

Relaciones dentro de la Institución: vínculo que se establece entre cuidadores y profesionales, con los niños, con sus familias de origen y con el ICBF.

Prácticas institucionales de cuidado: narraciones de cuidadores y profesionales sobre sus actividades diarias (esta categoría se acompaña de observaciones).

\section{Subcategorías emergentes de entrevista}

1. Cambio de formas de relación.

2. Características de la población atendida.

3. Necesidades de NNA.

Se tiene en cuenta la subcategoría: relación entre niños y cuidadores/profesionales.

No existen subcategorías. Se les preguntaba a cuidadores/profesionales tanto por su rutina diaria como por experiencias significativas vividas con NNA.

\section{Resultados}

\section{Cambios a lo largo de la historia institucional}

Con base en el análisis documental y en las entrevistas realizadas a directivos de la institución, se evidencian una serie de cambios, a lo largo de la historia institucional.

Desde su fundación en 1959 hasta 1997, la institución se organizaba alrededor de la figura de su fundadora quien representaba una figura materna ${ }^{9}$ para los NNA acogidos, de acuerdo con criterios discrecionales. A partir de 1970, el ICBF empieza a apoyar económicamente a la institución; en 1992 la entidad estatal les envía niños sin permitir procesos de selección. En 1996 ICBF requiere de licitación pública para continuar con los aportes. A partir de 2006, con la Ley 1098, la institución comienza a operar bajo los principios de protección integral y restablecimiento de derechos, abandonando la perspectiva de beneficencia. Esto visibilizó una tensión entre saberes expertos y saberes legos que, junto al uso de protocolos de atención y de herramientas burocráticas, puede leerse como medida de control o medida para el bienestar de los niños (Villalta, 2013). Actualmente quienes acceden con declaratoria de adoptabilidad han estado en otras instituciones de protección y han crecido escuchando un discurso que prioriza los derechos de los niños. Este discurso ha modificado la relación que establecen con la institución.

\footnotetext{
${ }^{9}$ La fundadora de la institución fue directora de la misma hasta su fallecimiento en el año 2009.
} 
El vínculo creciente de la institución con el ICBF propició la pérdida de la independencia económica, pasando de ser un hogar benefactor que voluntariamente acogía niños desprotegidos, a ser una institución prestadora de servicios del Estado, cuyo propósito es garantizar los derechos plenos de NNA. Actualmente la institución vela por el bienestar integral de los niños y la mayoría de ellos reconocen que les brinda lo que en sus casas no tenían'10. Dos empleados de la institución son egresados de la misma; algunos de los adolescentes internos laboran con el ICBF, mientras culminan sus estudios universitarios y otros, respaldados por esta institución, han recibido reconocimientos deportivos en eventos locales y nacionales.

\section{Concepciones de niños y adolescentes y tipos de relación}

Durante el trabajo de campo emergieron tres concepciones de niños enunciadas por las y los participantes: 1) hijos de Bienestar, 2) niños en carencia, 3) niños resilientes. Esta organización permite comprender las formas según las cuales profesionales y cuidadores entienden y se relacionan con NNA. A su vez, las categorías emergentes tienen que ver con formas de relación y de prácticas diarias establecidas entre profesionales o cuidadores y niños (figura 1). Si bien puede haber una concepción de un niño como hijo de Bienestar y un tipo de relación que privilegia un vínculo educativo, este puede estar acompañado de otros tipos de relación en la práctica.

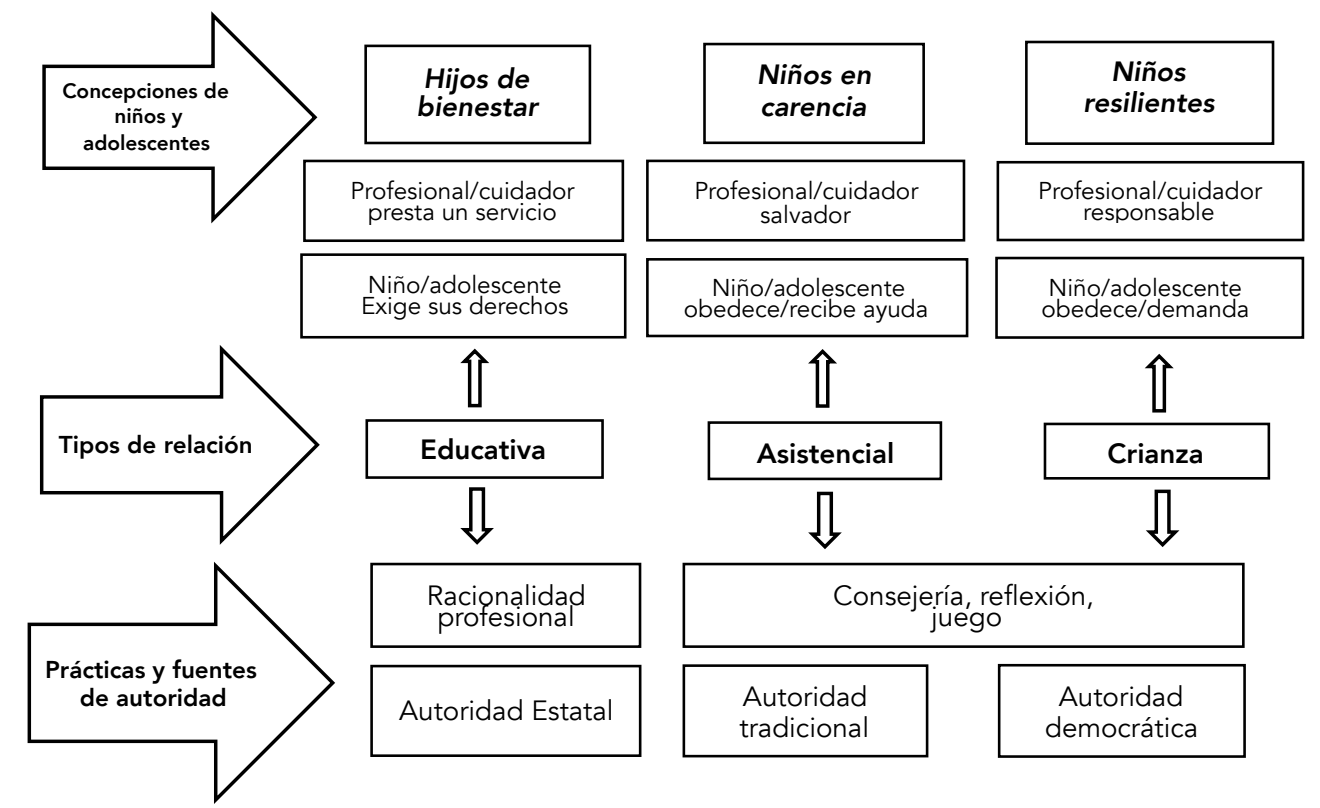

Figura 1. Concepciones de niños y tipos de relación

1o Los NNA señalan la posibilidad de participar en actividades recreativas, el apoyo escolar y el trato respetuoso que obtienen de cuidadores y profesionales. 
A continuación, se describirá cada categoría emergente y sus relaciones.

\section{Hijos de Bienestar}

Se encontró que algunos de los profesionales nombran a los niños como «hijos de Bienestar»11. Esta denominación se relaciona con la proclamación (a través de la CIDN en 1991) de los niños como sujetos de derechos y de especial protección por parte de las y los adultos.

(...) son Hijos de Bienestar (...) ellos crecieron en un medio donde todo el tiempo se les estaba diciendo: existe el interés superior del niño, la prevalencia, los derechos del niño y los derechos de los adolescentes... el proceso de internado en el que ellos crecen es un proceso donde ellos no tienen muchos deberes y no son muy autónomos (...) Los muchachos saben que Bienestar Familiar da unos recursos por ellos; entonces ellos exigen y creen que tienen derechos y no tienen obligaciones. (Profesional, 2016)

Son hijos de Bienestar porque quienes los representan son defensores de familia - trabajadores del Estado-. Si se presenta algún inconveniente entre los NNA y sus cuidadores, algunos optan por comunicarse con su defensor o defensora de familia. Por otra parte, existen limitaciones al requerimiento de deberes por parte de los profesionales hacia los adolescentes. Por ejemplo, los profesionales de la institución insisten en que los mayores -14 años en adelante- deberían aprender a preparar sus alimentos, pero esta acción no es posible porque para ello se precisa de prácticas cotidianas en el entorno familiar, las cuales se encuentran restringidas en la institución. Habilidades básicas como la mencionada son necesarias para la vida autónoma y se facilitan en un entorno familiar.

Según los profesionales, en los niños considerados como hijos de Bienestar se presenta una dificultad para asumir la norma: quienes los cuidan en la institución de protección están desprovistos de la autoridad derivada de una relación filial.

He tenido la oportunidad de sentarme con varios a darles consejos; algunos me cuentan sus problemas. No todos, mientras que hay otros, pues los más difíciles —digámoslo así-, los reacios. «No me diga porque, ¿usted quién es? Usted no es mi mamá, usted es una aparecida», como una vez me dijo un chico. (Cuidadora, 2016)

${ }^{11}$ Alude al Instituto Colombiano Bienestar Familiar. 
Las dificultades con el manejo de la norma se presentan, principalmente, entre los adolescentes y quienes los cuidan, porque permanecen más tiempo con ellos. Con los profesionales dichos inconvenientes no son frecuentes.

Existen más dificultades con las figuras de autoridad que son los cuidadores; con nosotros [profesionales de apoyo] realmente no hay tanta confrontación; lo que hago es apoyar para que se establezca la alternativa de restauración. (Profesional, 2016)

Esta concepción de hijo de Bienestar se relaciona no solo con la actitud cuestionadora que asumen diferentes NNA frente a las normas, sino con la asunción de comportamientos utilitaristas en palabras de las y los profesionales. Estos expresan que los NNA, al conocer sus derechos, hacen un uso utilitario o estratégico de ello para obtener beneficios (como llamar a su defensor de familia cuando tienen un malentendido con sus cuidadores o acceder a permisos), sin seguir el conducto regular de la institución.

Ellos tienen claro el rol que desempeñan los profesionales aquí en la institución; ellos saben que, de acuerdo a sus intereses, así mismo van buscando a la persona. (Cuidador, 2016)

Este uso utilitarista del discurso del interés superior del niño para exigir un trato diferente puede leerse como un acto performativo, desde lo propuesto por Llobet (2011), o un fenómeno semejante al uso estratégico de una identidad planteado por SánchezReyes (2015). Por ejemplo, los adolescentes en ocasiones manejan como recurso de poder frente a profesionales de psicología o trabajo social la firma en la asistencia a actividades, la cual es requerida por la entidad estatal para corroborar que la institución de protección está cumpliendo con su labor.

Ellos [la entidad estatal] vienen y revisan las carpetas, «muéstreme la firma de ellos»12. Entonces ellos [los adolescentes] todavía lo usan a uno con eso; no quieren firmar o quieren hacerse los locos. (Profesional, 2016)

Finalmente, la concepción de niño como hijo de Bienestar y la lectura que los NNA hacen de la institución como una entidad garante de sus derechos, se encuentra en tensión con la labor de educar que ejercen los cuidadores. La labor de educar implica para los profesionales el cumplimiento de las normas de la institución por parte de los niños,

${ }^{12}$ Los NNA deben firmar asistencia después de su participación en las actividades. 
mientras que la labor de garantizar derechos implica para los niños que los profesionales son reconocidos como prestadores de un servicio, más que como figuras de autoridad.

La institucionalidad les exige tanto a profesionales como a cuidadores el cumplimiento de procesos técnico-burocráticos y delimita profesionalmente sus funciones, lo que configura relaciones caracterizadas por la formalidad, mientras que en las familias los roles de padre o madre no están escritos y las labores de ambos, al igual que la forma de relación con sus hijos, suceden de forma más espontánea.

Un día cotidiano de trabajo es llegar a las 8 de la mañana, estar siempre con muchas cosas pendientes, estar en función de hablar, de hacer seguimiento a los muchachos, pero también de hacer reuniones de equipo para las dificultades que se presentan en la cotidianidad a nivel disciplinario o de cualquier tipo. (Profesional, 2016)

Sin embargo, los profesionales y cuidadores reconocen que dentro de las necesidades de los niños institucionalizados hay un pedido de afecto, fundamental para su constitución como seres humanos ${ }^{13}$, que no aparece dentro del procedimiento porque es un pedido usualmente adherido al espacio familiar. Por lo tanto, en la práctica diaria de profesionales y cuidadores aparece una tensión entre los pedidos institucionales y los pedidos de los niños, lo que entorpece el establecimiento de relaciones de confianza.

Finalmente, la concepción de sujeto como hijos de Bienestar es compartida principalmente por profesionales y directivos de la misma. De parte de los directivos existe, además, una queja por la necesidad de generar agradecimiento frente a la institución por la labor impartida, como antaño sucedía cuando su fundadora estaba a cargo. Uno de los directivos relata que antes la relación era de beneficencia:

Ahora no, ahora le tienen que dar y más «como yo lo quiera y como yo lo pida». Entonces es romper eso y formarlos a que sean agradecidos.

Esta relación de agradecimiento no se da necesariamente porque, para algunos NNA, la institución solo debe garantizar sus derechos. El tipo de relación que prevalece es educativa, en tanto las prácticas de profesionales y cuidadores se rigen por una racionalidad profesional.

13 Diversos autores concuerdan en la importancia del establecimiento de un vínculo con otros para la constitución humana (Dolto, 1985; Spitz, 1969, Stern, 2010). 


\section{Niños en carencia}

Algunos profesionales plantean una concepción del niño institucionalizado como un sujeto en déficit por haber sido abandonado por su familia. Esta se relaciona con la búsqueda de afecto que, de acuerdo con las y los entrevistados, emprenden los NNA institucionalizados. Situación que se agudiza cuando los adolescentes están a punto de egresar.

Ellos tienen un vacío de abandono que es una cosa que casi no se llena, que tú te sientas abandonado o dejado de tu familia yo creo que es una situación que marca la vida de uno por siempre. (Profesional, 2016)

Este tipo de concepción de niño como carente conlleva una contraparte de cuidador o profesional visto como salvador o salvadora. A su vez, genera una deuda simbólica de los NNA hacia la institución. Lo anterior favorece a que, quienes se ubican bajo esta concepción, sean sumisos y receptivos frente a aquello que se les ofrece, lo que conlleva a ser considerados agradecidos, tal como sucedía cuando la institución era un hogar de acogida que propiciaba las prácticas caritativas descritas por Castro (2008). La asistencia social ejercida desde la caridad favorecía la constitución de la relación salvador/benefactor entre los adultos responsables del cuidado y los niños.

Esta concepción de NNA carentes hace que las y los adultos señalen la necesidad de afecto presente en los mismos, acompañada de una imposibilidad de la institución por suplir a cabalidad este pedido de afecto de los niños, función que se le otorga a la familia.

Para mí son niños de un hogar; igual nosotros como institución jamás vamos a reemplazar un hogar, con su mamá y su papá, pero sí hacemos que ellos sientan que aquí se les brinda el apoyo, son escuchados, se les da el amor. (Cuidadora, 2016)

Aunque cuidadores y profesionales afirman que la institución no es asimilable a un hogar, algunos plantean que puede brindar apoyo emocional a los NNA. Se identifica en las prácticas adoptadas un estilo más ligado a concepciones tradicionales de crianza, en donde se reconoce la necesidad del afecto de los niños y la posibilidad de ofrecer afecto por parte de los profesionales y cuidadores:

A nadie le perturba ser querido; los niños buscan ese afecto (...) quieren llamar la atención, lo que sea. (Cuidadora, 2016) 
Por otra parte, aquellos profesionales y cuidadores que enunciaron una concepción de niño en carencia, privilegian una relación de tipo asistencial que se ocupa principalmente de cubrir necesidades físicas básicas. Aunque también se evidencian prácticas que buscan hacer reflexionar a los niños presentes tanto en esta categoría como en la de niños resilientes. Una forma de hacer reflexionar, se presenta a continuación:

«Profesora mire que me miraron», es como llamando la atención, «¿Sí?, miłjo, ¿qué le pasó?» Entonces al llamarlo, «venga, hablemos, ¿qué le pasa?, ¿por qué se está portando así?» (Cuidadora, 2016)

En la concepción niños en carencia coincidían principalmente cuidadores y un directivo de la institución. Sin embargo, las prácticas de los profesionales difieren. Por ejemplo, no todos ellos responden a los pedidos de afecto de los niños una vez los identifican. En ocasiones interponen una barrera porque no quieren generar apego por parte de los niños.

\section{Niños resilientes ${ }^{14}$}

Los profesionales y cuidadores se refieren a la capacidad presente en los NNA institucionalizados para superar el sentimiento de abandono y construir un futuro. Situación que es posible cuando los cuidadores sienten que, a partir de su relación con los NNA y con el acompañamiento diario que les brindan, logran asemejar en algo lo que los padres realizan con sus hijos.

Son muchachos que generalmente uno los puede ver contentos, que se proyectan positivamente en el futuro para alcanzar sus metas y que esas experiencias no los definieron. (Cuidadora, 2016)

Cuando se les pregunta a los cuidadores por las estrategias que usan para orientar a los NNA, algunos relatan que hacen lo que ya les ha funcionado con sus propios hijos e hijas: una cuidadora descubrió que, desde que su hija practica natación, ha mejorado su desempeño académico; por esta razón incrementó las prácticas deportivas con los adolescentes a su cargo.

I4 Niños resilientes es una expresión empleado por los participantes, aunque no alude necesariamente al concepto de resiliencia. 
Hay profesores que son más que profesores; como amigos, como con una imagen más cercana a la paterna. Porque uno dice: «todos estos cuidadores y los profesionales son como tus padres». Falso. No todos crean el vínculo. (Profesional, 2016)

La creación de este vínculo se torna difícil para los NNA institucionalizados cuando hay alta rotación de personal (que no es el caso de la institución estudiada) o cuando los adolescentes se evaden de la institución ${ }^{15}$. Quienes se evaden, no son considerados por la mayoría de las y los entrevistados como resilientes.

Uno de los profesionales relató la difícil situación que vivió años atrás cuando uno de los adolescentes se evadió para ir a visitar a sus familiares y fue asesinado a los pocos días por una bala perdida en el barrio donde vivía su familia. Este tipo de situaciones atípicas son difíciles de superar por parte de cuidadores y profesionales que se comprometen con los proyectos de vida de NNA.

[Sobre su cambio de ser docente escolar a ser cuidadora] ya es pasar de tener un hijo a tener 33 hijos, donde nosotros tenemos que ver: que se cepillen, laven, tiendan, asistan, madruguen, sean ahorrativos, cumplan los horarios; bueno, todo lo que hace la mamá. (Cuidadora, 2016)

Los relatos que priorizan relaciones cercanas a las que se darían en las familias, enfatizan además en la necesidad de acompañar a los NNA en sus actividades cotidianas.

A mí me toca la parte de que ellos tengan un descanso [turno en la noche] donde estamos pendientes de una parte de sus bienes, de que alisten sus uniformes para el otro día ir al colegio, de que ya hayan finalizado sus tareas. (Cuidador, 2016)

Al respecto, una profesional y un cuidador reconocieron la necesidad de cercanía con los niños, pero plantearon que toman distancia frente a un rol paternal. La profesional de apoyo comentó no tener tiempo por la cantidad de trabajo administrativo que debe hacer, mientras que el cuidador relató lo siguiente:

Aparentemente solo el hecho de estar en las actividades deportivas y recreativas ha creado como ese vínculo especial (...) como esa relación digamos paternal, la cual uno trata de

\footnotetext{
15 Este hecho, poco frecuente, afecta el trabajo de profesionales y cuidadores dado que se preguntan: ¿por qué los NNA deciden retornar a hogares en donde sus derechos fueron vulnerados? Lo anterior merece otras indagaciones en estudios futuros.
} 
mantener como retirada, porque ellos van a sufrir algún día la decepción de que uno se tiene que ir o que ellos se van para otra parte. (Cuidador, 2016)

Se encuentra que en este tipo de concepción de sujeto como niño resiliente puede propiciar, en mayor medida, la creación de vínculos de confianza con los NNA atendidos. El establecimiento de un vínculo de confianza requiere tomar distancia de las labores burocráticas y posibilita el cumplimiento de la norma desde el respeto y el reconocimiento del otro.

Para algunos profesionales y cuidadores este modo resiliente de responder de los niños ante las adversidades, los lleva a conseguir logros académicos y deportivos. Los niños dentro de esta categoría son ubicados como sujetos obedientes de la norma, que demandan ayuda de parte de la institución para desarrollar sus actividades.

\section{Discusión}

Los resultados de investigación aportan en la comprensión de los tipos de relación y las concepciones de niño que se dan al interior de una institución de protección. Se expusieron tres diferentes concepciones de NNA enunciadas por los cuidadores o profesionales de la institución de protección estudiada: hijos de Bienestar, niños en carencia $y$ niños resilientes. Estas concepciones inciden en diferentes formas de relación y de prácticas diarias establecidas entre profesionales o cuidadores y niños. Así, la concepción de niño como hijo de Bienestar se relaciona con un vínculo educativo; la concepción de niño como carente se relaciona con una relación asistencial; y la concepción de niño como resiliente se vincula con una relación de crianza ${ }^{16}$.

La concepción hijos de Bienestar estaría relacionada con la perspectiva garantista y de restitución de derechos que promueve la legislación de protección de la infancia. El vínculo educativo que se construye entre los cuidadores y los NNA a partir de esta concepción se basa en la racionalización de la relación entre un sujeto demandante y otro que oferta contenidos y saberes, eclipsando las dimensiones afectivas de la relación. Este tipo de vínculo propicia, por un lado, una demanda insaciable o exigencia permanente

\footnotetext{
16 Según Levine y White (1987) por relación de crianza se entiende que el adulto cuidador o profesional de apoyo se asume como responsable del proceso de cuidado, crecimiento y, socialización de los NNA con el fin de propiciar que los estos se desarrollen de forma integral, integrándose a su contexto social y cultural.
} 
por parte de los NNA hacia la institución y, por otro, la expectativa de gratitud y éxito, de parte de la institución, en los procesos de inserción escolar y social.

La asunción por parte del Estado de la labor de crianza de niñas y niños abandonados por sus progenitores no solo requiere del establecimiento de vínculos de afecto, sino que precisa de límites para niños y niñas que tradicionalmente se han dado en espacios de socialización familiares, pero que se dificultan en espacios como las instituciones de protección en donde el discurso de restitución de derechos puede invisibilizar la necesidad de regulación que se requiere en la crianza de niños y niñas.

La concepción niño carente estaría avalada por varios autores que subrayan que la familia se constituye como un derecho fundamental y necesario para el desarrollo sano de los niños y niñas; y que su privación genera un estado de vacío (Villalobos, 2003), aislamiento social, desarraigo e incertidumbre frente a su futuro (Durán \& Valoyes, 2009). Desde esta perspectiva, su función no sería sustituible por otra institución (Rodrigo \& Palacios, 1998).

Valdría la pena preguntarse hasta qué punto la concepción de niño carente contribuye a formar ciudadanos con posibilidades de agencia y no a revictimizar a los niños o a legitimar una concepción de niños institucionalizados en déficit al no tener una familia que los acoja. Para aquellos niños que tienen como única alternativa el crecimiento dentro de una institución de protección resulta trascendental un cambio en la concepción que se ha generado frente a ellos por parte de la sociedad, de manera que se rescate su lugar de agentes y constructores de su realidad, en la vía que mencionan Obando et al. (2010). La concepción de niño carente se relaciona con el paradigma de la beneficencia y la caridad, según Castro (2008).

En la misma vía que mencionan Obando et al. (2010) se encuentra que la concepción de sujeto como niño resiliente puede propiciar, en mayor medida, la creación de vínculos de confianza con los NNA atendidos. El establecimiento de un vínculo de confianza requiere tomar distancia de las labores burocráticas y posibilita el cumplimiento de la norma desde el respeto y el reconocimiento del otro. La concepción de niño resiliente está relacionada con la noción de niño sujeto de derechos, haciendo énfasis en el rol activo de los niños como ciudadanos con posibilidad de transformar su realidad y construir un proyecto de vida.

Esta concepción, que permite destacar las posibilidades de agencia de los niños institucionalizados, se relaciona con la creación de vínculos de apoyo o afectivos con 
personas ajenas a la familia dentro del marco institucional. Lo anterior requiere del reconocimiento por parte del Estado de que la labor de restablecimiento de derechos a niños y niñas no se logra solamente por medio de la aplicación de protocolos de atención, sino que implica la generación de vínculos significativos con los cuidadores y profesionales a cargo.

Sin desconocer que el Estado debe garantizar el derecho a la familia a niños y niñas, y que la institucionalización no es la alternativa más deseable para hacer frente a la problemática de vulneración de derechos y abandono de la niñez, se señala que las funciones actuales de la familia se derivan de un proceso sociohistórico. La asunción de la socialización de niños y niñas por parte de la familia como única institución encargada es puesta en perspectiva cuando se encuentra que existen instituciones de protección (como la estudiada) que asumen esta misma labor posibilitando que sus egresados se integren a la vida en sociedad.

\section{Referencias}

Amar, J. J., Tirado, D., \& Barreneche, A. (2007). Impacto de los programas de bienestar en la superación de la pobreza y el desarrollo de los niños en Colombia. Investigación y Desarrollo, 15(1), 124-149.

Ariès, P. (1987). El niño y la vida familiar en el antiguo régimen. Madrid: Taurus.

Castrillón, M. C. (2008). ¿Menores ciudadanos o sujetos de derechos tutelados? Discursividades institucionales sobre los derechos de la niñez y la adolescencia en Brasil y Colombia. Campos, Revista de Antropología Social, 9(1), 29-50. https://doi.org/10.5380/ cam.vgi1.13868

Castro, B. (2008). Los inicios de la asistencia social en Colombia. Revista CS, (1), 157-188. https://doi.org/10.18046/recs.i1.405

Comisión Interamericana de Derechos Humanos. (2013). El derecho niño y la niña a la familia. Cuidado alternativo. Poniendo fin a la institucionalización en las Américas (Informe Oficial de la OEA Doc. 54). Nueva York: Autor, Unicef.

Crotty, M. (2006). The foundations of social research. Londres: Sage.

Deambrosio, M., Gutiérrez de Vázquez, M., Arán-Filippetti, V., \& Román, F. (2018). Efectos del maltrato en la neurocognición: un estudio en niños maltratados institucionalizados y no institucionalizados. Revista Latinoamericana de Ciencias Sociales, Niñez y Juventud, 16(1), 239-253. https://doi.org/10.1160o/1692715x.16114 
Dolto, F. (1985). En el juego del deseo. Buenos Aires: Paidós.

Durán E., \& Valoyes, E. (2009). Perfil de los niños, niñas y adolescentes sin cuidado parental en Colombia. Revista Latinoamericana de Ciencias Sociales, Niñez y Juventud, $7(2), 761-783$.

Fernández-Daza, M. P. (2018). El acogimiento familiar en Iberoamérica. Saúde e Sociedade, 27, 268-289. http://dx.doi.org/10.1590/s0104-12902018170647

Fernández-Daza, M. P., \& Fernández-Parra, A. (2013). Problemas de comportamiento y competencias psicosociales en niños y adolescentes institucionalizados. Universitas Psychologica, 12(3), 797-810. https://doi.org/10.11144/javeriana.upsy12-3.pccp

Gabatz, R. I. B., Schwartz, E., Milbrath, V. M., Carvalho, H. C. W. D., Lange, C., \& Soares, M. C. (2018). Formation and disruption of bonds between caregivers and institutionalized children. Revista Brasileira de Enfermagem, 71, 2650-2658. https://doi.org/ 10.1590/0034-7167-2017-0844

Galeano, M. E. (2004). Estrategias de investigación social cualitativa. Medellín: La Carreta.

Grinberg, J. (2015). Entre la pediatría, el psicoanálisis y el derecho: apuntes sobre la recepción, reelaboración y difusión del «maltrato infantil» en Argentina. Revista de Estudios Sociales, (53), 77-89. https://doi.org/10.7440/res53.2015.06

Instituto Colombiano de Bienestar Familiar. (2015, octubre 7). Cerca de 5 mil niños y niñas esperan para ser adoptados. [Comunicado de prensa]. Recuperado de: https://www.icbf.gov.co/sites/default/files/comunicado2-07-10-2015.pdf

Jiménez, D. (2009). Responsabilidad penal juvenil en Colombia: de la ideología tutelar a la protección integral. Diálogos de Derecho y Política, (1), 7-17.

Jorge, E., \& González, C. (2017). Estilos de crianza parental: una revisión teórica. Informes Psicológicos, 17(2), 39-66. https://doi.org/10.18566/infpsic.v17n2ao2

Levin, E. (2000). La función del hijo: espejos y laberintos de la infancia. Buenos Aires: Nueva Visión.

Levine, R. A., \& White, M. I. (1987). El hecho humano: bases culturales del desarrollo educativo. Madrid: Visor.

Llobet, V. (2011). Las políticas para la infancia y el enfoque de derechos en América Latina: algunas reflexiones sobre su abordaje teórico. Fractal: Revista de Psicología, 23(3), 447-46o. http://dx.doi.org/10.1590/S1984-02922011000300002

Micolta, A. (2011). Apuntes históricos de la paternidad y la maternidad. Prospectiva. Revista de Trabajo Social e Intervención Social, (13), 89-121. https://doi.org/10.25100/ prts.voi13.1179 
Nogueira, A. (2017). La protección convencional de los Derechos de los Niños y los estándares de la corte IDH sobre medidas especiales de protección por parte de los Estados partes respecto de los niños, como fundamento para asegurar constitucionalmente los Derechos de los niños y adolescentes. Ius et Praxis, 23(2), 415-462. http://dx.doi.org/10.4067/So718-00122017000200415

Obando, O. L., Villalobos, M. E., \& Arango, S. L. (2010). Resiliencia en niños con experiencias de abandono. Acta Colombiana de Psicología, 13(2), 149-159.

Otálvaro, J., Peñaranda, F., Bastidas, M., Torres, N., \& Trujillo, J. (2016). Reformas neoliberales y sus implicaciones en un programa de apoyo a la crianza: el caso de los hogares comunitarios Familia, Mujer e Infancia en Colombia. Revista Latinoamericana de Ciencias Sociales, Niñez y Juventud, 14(1), 645-658.

Palummo, J. (2013). La situación de niños, niñas y adolescentes en las instituciones de protección y cuidado de América Latina y el Caribe. Panamá: Unicef.

Paz, A. L. (2014). La intervención social y sus retos frente al quehacer de las ciencias sociales. En Grupo de Investigación en Intervención y Responsabilidad Social (Ed.), Intervención social y el debate sobre lo público: reflexiones conceptuales y casos locales (91-114). Cali: Editorial Universidad Icesi.

Rodrigo, M. J., \& Palacios, J. (Coords.) (1998). Familia y desarrollo humano. Madrid: Alianza.

San Martín, D. (2014). Teoría fundamentada y Atlas.ti: recursos metodológicos para la investigación educativa. Revista Electrónica de Investigación Educativa, 16(1), 104-122. Recuperado de: http://redie.uabc.mx/vol16no1/contenido-sanmartin.html

Sánchez-Reyes, J. E. (2015). Identidad étnica de estudiantes Misak de la Universidad del Valle. (Tesis de maestría no publicada). Universidad del Valle, Cali, Colombia.

Santillán, L. (2009). La crianza y educación infantil como cuestión social, política y cotidiana: una etnografía en barrios populares del Gran Buenos Aires. Anthropologica, 27(27), 47-74.

Silva da Cruz, E. J., Dias, G. B, \& da Silva J. P. (2014). Estudo do «Ages and Stages Questionnaires» com cuidadores de crianças institucionalizadas. Psico-USF, 19(3), 411-420. http://dx.doi.org/10.1590/1413-82712014019003004

Smyke, A. T., Koga, S. F., Johnson, D. E, Fox, N. A., Marshall, P. J., Nelson, C. A., \& Zeanah, C. (2007). The caregiving context in institution-reared and family-reared infants and toddlers in Romania. Journal of Child Psychology \& Psychiatry, 48(2), https://doi.org/10.1111/j.1469-7610.2006.01694.x

Spitz, R. (1969). El primer año de vida del niño. Barcelona: Fondo de Cultura Económica. 
Stern, D. (2010). Forms of vitality: Exploring dynamic experience in psychology and the arts. Oxford: Oxford University Press.

Strauss, A. \& Corbin, J. (2002). Bases de la investigación cualitativa: técnicas y procedimientos para desarrollar la teoría fundamentada. Medellín: Editorial Universidad de Antioquia

Villalobos, M. (2003). Factores implicados en el proceso de ruptura de la resiliencia, medios traumatizantes y procesos de resiliencia. Coloquio Internacional Universidad de Nancy, Nancy, Francia.

Villalta, C. D. (2013). Un campo de investigación: las técnicas de gestión y los dispositivos jurídico-burocráticos destinados a la infancia pobre en la Argentina. Civitas, 13(2), 235-258. https://doi.org/10.15448/1984-7289.2013.2.15482

Villar, R. (2001, septiembre). La institucionalidad política y el tercer sector en Colombia: notas para una reflexión. Ponencia presentada en III Encuentro de la Red Latinoamericana y del Caribe ISTR-LAC, Buenos Aires, Argentina. 